\title{
Shelf Life Evaluation of Biscuits and Cookies Incorporating Germinated Pumpkin Seed Flour
}

\author{
Neeta Kumari $^{1 *}$, Sangeeta C. Sindhu ${ }^{1}$, Varsha Rani ${ }^{1}$ and Varsha Kumari ${ }^{2}$ \\ ${ }^{I}$ Department of Foods and Nutrition, I.C. College of Home Science \\ CCS Haryana Agricultural University, Hisar-125001, Haryana, India \\ ${ }^{2}$ KVK Vaishali, DRPCAU, Pusa (Bihar), India \\ *Corresponding author
}

\section{A B S T R A C T}

\begin{tabular}{l} 
K e y w o r d s \\
Pumpkin seeds, \\
Biscuits, Cookies, \\
$\begin{array}{l}\text { Sensory evaluation, } \\
\text { Shelf life, Peroxide } \\
\text { value, Total plate } \\
\text { count }\end{array}$ \\
\hline $\begin{array}{l}\text { Article Info } \\
\text { Accepted: } \\
\text { 12 December } 2020 \\
\text { Available Online: } \\
\text { 10 January 2021 }\end{array}$ \\
\hline
\end{tabular}

\section{Introduction}

Pumpkin seeds are locally available, underutilized but potential food sources. The most used part of the pumpkin fruit is the flesh, which is used as a vegetable in food preparations. Pumpkin seeds are generally discarded during processing and are generally regarded as a waste. Pumpkin seeds can play important role in food and health industry. They can be consumed regularly without causing any side effects on human health (Maheshwari et al., 2015). Consumption of diets rich in pumpkin seeds also have been associated to lower the chances of occurrence of many types of cancer like colorectal, lung, breast, gastric cancer (Huang et al., 2004). These are loaded with nutrients and medicinal properties. Pumpkin seeds are rich natural source of protein (25 to $37 \%$ ) and oil (37 to $45 \%$ ). In addition, they are also good sources of minerals, dietary fibre, health-benefiting vitamins and mono-unsaturated fatty acids, which are good for heart health (Khairi et al., 2014). 
Rightly termed as nutritional powerhouse, as these seeds are excellent nutrient source filled with minerals mainly zinc, phosphorous, magnesium, potassium and selenium responsible for fighting diseases and can act as weapon for fighting diseases such as arthritis, inflammation, prostate cancer etc. (Patel and Rauf, 2017). They are also rich source of zinc, lignans, and phytosterols such as delta 7-sterols and delta 5-sterols essential amino acid like as tryptophan and glutamate and are beneficial for maintenance of immune system, cell growth and multiplication, eye and skin health, insulin regulation and male sexual functions such as sperm generation and testosterone metabolism (Montesano et al., 2018; Karrar et al., 2019).

Pumpkin seeds contain 31.48 per cent crude fiber (Nyam et al., 2013). Fiber present in pumpkin seeds can prevent constipation, diabetes, prolong intestinal transit time, lower cholesterol level and provide satiety. Pumpkin seed flour can be used to fortify soups, cookies, pancakes and breads. Moreover, it is also used to fortify wheat flour to produce bakery products like pastries with unique and nutty taste (Lestari and Meiyanto, 2018).

Value addition of existing foods like biscuits and cookies with such ingredients is a simple and feasible way of enhancing nutritional values of foods and add variety to snacking with health.

\section{Materials and Methods}

\section{Product development}

Present study was carried out in Department of Foods and Nutrition, I.C. College of Home science, Chaudhary Charan Singh Haryana Agricultural University, Hisar. Pumpkins were procured from the local market and seeds were separated from the pulp.
For the preparation of biscuits and cookies the required ingredients namely, whole wheat flour, refined wheat flour, ghee, milk, refined sugar, ammonia and baking powder were purchased in a single lot from the local market of Hisar. Three types of cookies and whole wheat flour biscuits were developed i.e. Type I, Type II and Type III, respectively, by replacing 10, 20 and 30 per cent of flour with germinated pumpkin seed flour (Kumari and Sindhu, 2019; Kumari et al., 2020).

\section{Shelf- life evaluation}

Value added products were stored at room temperature in polythene zipper bag (LD-PE, clear zip lock, thickness $0.11 \mathrm{~mm}$ ) and drawn at interval of 15 days of storage for 75 days. These were evaluated for various sensory characteristics i.e. colour, appearance, aroma, texture, taste and overall acceptability using 9 point hedonic scale by a panel of 10 semijudge from the Department of Foods and Nutrition, I.C. College of Home Science. Peroxide value was determined by the method of AOAC (2010). Total plate count was recorded as per the procedures by APHA (1980). using Nutrient Agar for total plate count.

\section{Results and Discussion}

\section{Sensory characteristics}

The whole wheat flour biscuits could be stored successfully for 60days whereas cookies were acceptable at $75^{\text {th }}$ day also (Table1\&2).

\section{Peroxide value}

Table 3 presents the peroxide value (meq peroxide/1000g) of developed value added whole wheat flour biscuits during the storage period. Peroxide value significantly $(\mathrm{P} \leq 0.05)$ increased with the period of storage from day 
$15^{\text {th }}$ to $75^{\text {th }}$ day. In Type-I, Type-II and TypeIII biscuits, peroxide value ranged from 4.27 to 6.33 (Type-I), 3.20 to 5.03 (Type-II) and 2.81 to 4.47 (Type-III) meq peroxide/1000g.

During storage of cookies (Table 4) peroxide value of control cookies ranged from 3.20 to 5.00 meq peroxide $/ 1000 \mathrm{~g}$ ( $15^{\text {th }}$ to $75^{\text {th }}$ days). Peroxide value during the period $15,30,45$,
60 and $75^{\text {th }}$ days were $2.40,3.30,3.40,4.27$ and 4.60 meq peroxide $/ 1000 \mathrm{~g}$ for Type-I cookies. A significant $(\mathrm{P} \leq 0.05)$ difference was observed after $45^{\text {th }}$ day of storage. The peroxide value of Type-II and Type-III cookies varied from 2.27 ( $15^{\text {th }}$ day) to 4.13 $\left(75^{\text {th }}\right.$ day $)$ meq peroxide $/ 1000 \mathrm{~g}$ and $1.80\left(15^{\text {th }}\right.$ day) to $3.70\left(75^{\text {th }}\right.$ day $)$ meq peroxide $/ 1000 \mathrm{~g}$, respectively.

Table.1 Organoleptic acceptability of developed value-added whole-wheat flour biscuits during storage

\begin{tabular}{|c|c|c|c|c|c|c|}
\hline \multirow{2}{*}{$\begin{array}{c}\text { Level of } \\
\text { incorporation }\end{array}$} & \multicolumn{6}{|c|}{ Storage period (days) } \\
\hline & 15 & 30 & 45 & 60 & 75 & $\begin{array}{c}\text { C.D } \\
(\mathbf{P} \leq \mathbf{0 . 0 5})\end{array}$ \\
\hline \multicolumn{7}{|l|}{ Colour } \\
\hline Control & $8.80 \pm 0.13$ & $8.20 \pm 0.29$ & $8.20 \pm 0.29$ & $8.00 \pm 0.26$ & $5.90 \pm 0.46$ & 0.87 \\
\hline Type-I & $8.60 \pm 0.16$ & $7.90 \pm 0.10$ & $7.90 \pm 0.10$ & $7.90 \pm 0.10$ & $5.60 \pm 0.56$ & 0.78 \\
\hline Type-II & $8.10 \pm 0.18$ & $7.70 \pm 0.16$ & $7.60 \pm 0.15$ & $7.60 \pm 0.16$ & $4.80 \pm 0.59$ & 0.86 \\
\hline Type-III & $7.80 \pm 0.20$ & $6.80 \pm 0.27$ & $6.60 \pm 0.25$ & $6.60 \pm 0.27$ & $4.20 \pm 0.71$ & 1.10 \\
\hline \multicolumn{7}{|l|}{ Appearance } \\
\hline Control & $8.70 \pm 0.15$ & $8.20 \pm 0.29$ & $8.10 \pm 0.28$ & $7.60 \pm 0.27$ & $5.90 \pm 0.46$ & 0.87 \\
\hline Type-I & $8.60 \pm 0.16$ & $8.00 \pm 0.15$ & $7.70 \pm 0.21$ & $7.10 \pm 0.18$ & $5.50 \pm 0.52$ & 0.80 \\
\hline Type-II & $8.00 \pm 0.21$ & $7.50 \pm 0.22$ & $7.50 \pm 0.22$ & $7.60 \pm 0.22$ & $4.80 \pm 0.57$ & 0.93 \\
\hline Type-III & $7.60 \pm 0.22$ & $6.80 \pm 0.39$ & $6.80 \pm 0.29$ & $6.80 \pm 0.33$ & $4.10 \pm 0.66$ & 1.16 \\
\hline \multicolumn{7}{|l|}{ Aroma } \\
\hline Control & $8.60 \pm 0.22$ & $8.10 \pm 0.28$ & $7.80 \pm 0.13$ & $7.90 \pm 0.31$ & $5.60 \pm 0.37$ & 0.78 \\
\hline Type-I & $8.40 \pm 0.22$ & $7.80 \pm 0.13$ & $7.70 \pm 0.13$ & $7.60 \pm 0.16$ & $4.90 \pm 0.38$ & 0.65 \\
\hline Type-II & $8.10 \pm 0.23$ & $7.20 \pm 0.20$ & $7.30 \pm 0.21$ & $7.50 \pm 0.22$ & $4.20 \pm 0.51$ & 0.86 \\
\hline Type-III & $7.60 \pm 0.27$ & $6.60 \pm 0.37$ & $6.60 \pm 0.22$ & $6.70 \pm 0.37$ & $3.60 \pm 0.52$ & 1.04 \\
\hline \multicolumn{7}{|l|}{ Texture } \\
\hline Control & $8.60 \pm 0.22$ & $8.20 \pm 0.29$ & $7.70 \pm 0.21$ & $7.30 \pm 0.26$ & $5.50 \pm 0.37$ & 0.79 \\
\hline Type-I & $8.50 \pm 0.22$ & $7.80 \pm 0.13$ & $7.70 \pm 0.15$ & $7.10 \pm 0.28$ & $5.00 \pm 0.37$ & 0.70 \\
\hline Type-II & $8.00 \pm 0.21$ & $7.20 \pm 0.20$ & $7.40 \pm 0.16$ & $7.10 \pm 0.18$ & $4.40 \pm 0.50$ & 0.80 \\
\hline Type-III & $7.30 \pm 0.21$ & $6.20 \pm 0.42$ & $6.30 \pm 0.30$ & $6.10 \pm 0.35$ & $3.80 \pm 0.59$ & 1.13 \\
\hline \multicolumn{7}{|l|}{ Taste } \\
\hline Control & $8.60 \pm 0.22$ & $7.80 \pm 0.33$ & $7.30 \pm 0.21$ & $6.40 \pm 0.27$ & $5.00 \pm 0.47$ & 0.90 \\
\hline Type-I & $8.50 \pm 0.22$ & $7.40 \pm 0.16$ & $7.10 \pm 0.18$ & $6.70 \pm 0.21$ & $4.50 \pm 0.45$ & 0.76 \\
\hline Type-II & $8.30 \pm 0.30$ & $7.10 \pm 0.18$ & $6.70 \pm 0.21$ & $6.60 \pm 0.22$ & $3.70 \pm 0.47$ & 0.85 \\
\hline Type-III & $7.40 \pm 0.27$ & $6.00 \pm 0.39$ & $5.90 \pm 0.41$ & $5.80 \pm 0.25$ & $2.50 \pm 0.50$ & 1.07 \\
\hline \multicolumn{7}{|c|}{ Overall Acceptability } \\
\hline Control & $8.66 \pm 0.18$ & $8.10 \pm 0.28$ & $7.82 \pm 0.17$ & $7.44 \pm 0.21$ & $5.58 \pm 0.36$ & 0.71 \\
\hline Type-I & $8.52 \pm 0.17$ & $7.78 \pm 0.10$ & $7.62 \pm 0.07$ & $7.28 \pm 0.07$ & $5.10 \pm 0.39$ & 0.57 \\
\hline Type-II & $8.10 \pm 0.20$ & $7.30 \pm 0.17$ & $7.32 \pm 0.14$ & $7.28 \pm 0.14$ & $4.38 \pm 0.47$ & 0.74 \\
\hline Type-III & $7.54 \pm 0.20$ & $6.44 \pm 0.36$ & $6.48 \pm 0.24$ & $6.40 \pm 0.29$ & $3.64 \pm 0.55$ & 0.96 \\
\hline
\end{tabular}

Values are mean \pm SE of ten observations

Control=WWF: GPSF:: 100:0, Type-I=WWF: GPSF:: 90:10, Type-II=WWF: GPSF:: 80:20,

Type-III= WWF: GPSF:: 70:30, WWF: Whole wheat flour, GPSF: Germinated pumpkin seed flour 
Table.2 Organoleptic acceptability of developed value added cookies during storage

\begin{tabular}{|c|c|c|c|c|c|c|}
\hline \multirow{2}{*}{$\begin{array}{c}\text { Level of } \\
\text { incorporation }\end{array}$} & \multicolumn{6}{|c|}{ Storage (days) } \\
\hline & 15 & 30 & 45 & 60 & 75 & $\begin{array}{c}\text { C.D } \\
(\mathbf{P} \leq \mathbf{0 . 0 5})\end{array}$ \\
\hline \multicolumn{7}{|l|}{ Colour } \\
\hline Control & $8.20 \pm 0.20$ & $8.30 \pm 0.15$ & $7.80 \pm 0.15$ & $7.70 \pm 0.13$ & $6.70 \pm 0.15$ & 0.46 \\
\hline Type-I & $8.10 \pm 0.18$ & $8.40 \pm 0.16$ & $8.25 \pm 0.25$ & $7.60 \pm 0.16$ & $6.90 \pm 0.18$ & 0.54 \\
\hline Type-II & $8.10 \pm 0.10$ & $8.10 \pm 0.10$ & $8.30 \pm 0.26$ & $7.30 \pm 0.15$ & $7.50 \pm 0.22$ & 0.51 \\
\hline Type-III & $8.10 \pm 0.28$ & $7.80 \pm 0.13$ & $7.80 \pm 0.20$ & $6.70 \pm 0.26$ & $6.70 \pm 0.26$ & 0.66 \\
\hline \multicolumn{7}{|l|}{ Appearance } \\
\hline Control & $8.20 \pm 0.20$ & $8.00 \pm 0.15$ & $7.80 \pm 0.15$ & $7.70 \pm 0.13$ & $6.00 \pm 0.21$ & 0.48 \\
\hline Type-I & $8.20 \pm 0.13$ & $8.10 \pm 0.10$ & $8.20 \pm 0.25$ & $7.60 \pm 0.16$ & $6.30 \pm 0.26$ & 0.55 \\
\hline Type-II & $8.00 \pm 0.15$ & $7.90 \pm 0.10$ & $8.30 \pm 0.26$ & $7.20 \pm 0.13$ & $7.50 \pm 0.17$ & 0.49 \\
\hline Type-III & $8.30 \pm 0.26$ & $8.30 \pm 0.26$ & $7.80 \pm 0.20$ & $6.60 \pm 0.22$ & $6.80 \pm 0.25$ & 0.68 \\
\hline \multicolumn{7}{|l|}{ Aroma } \\
\hline Control & $8.20 \pm 0.20$ & $8.20 \pm 0.20$ & $7.90 \pm 0.10$ & $7.70 \pm 0.15$ & $6.80 \pm 0.25$ & 0.53 \\
\hline Type-I & $8.30 \pm 0.15$ & $8.30 \pm 0.15$ & $8.20 \pm 0.25$ & $7.40 \pm 0.16$ & $6.80 \pm 0.25$ & 0.57 \\
\hline Type-II & $8.00 \pm 0.15$ & $8.20 \pm 0.13$ & $8.20 \pm 0.33$ & $7.00 \pm 0.15$ & $6.40 \pm 0.22$ & 0.59 \\
\hline Type-III & $8.30 \pm 0.26$ & $8.30 \pm 0.26$ & $7.60 \pm 0.22$ & $6.30 \pm 0.15$ & $6.40 \pm 0.16$ & 0.62 \\
\hline \multicolumn{7}{|l|}{ Texture } \\
\hline Control & $8.10 \pm 0.18$ & $8.10 \pm 0.10$ & $7.80 \pm 0.13$ & $7.70 \pm 0.21$ & $7.00 \pm 0.21$ & 0.47 \\
\hline Type-I & $8.20 \pm 0.13$ & $8.10 \pm 0.10$ & $8.20 \pm 0.25$ & $7.30 \pm 0.15$ & $7.10 \pm 0.18$ & 0.49 \\
\hline Type-II & $7.90 \pm 0.18$ & $7.90 \pm 0.18$ & $8.40 \pm 0.27$ & $7.20 \pm 0.13$ & $7.10 \pm 0.23$ & 0.58 \\
\hline Type-III & $8.40 \pm 0.27$ & $8.00 \pm 0.15$ & $7.70 \pm 0.26$ & $6.40 \pm 0.16$ & $6.70 \pm 0.21$ & 0.62 \\
\hline \multicolumn{7}{|l|}{ Taste } \\
\hline Control & $8.10 \pm 0.18$ & $8.10 \pm 0.15$ & $7.70 \pm 0.31$ & $7.50 \pm 0.17$ & $7.20 \pm 0.29$ & 0.64 \\
\hline Type-I & $8.30 \pm 0.15$ & $8.20 \pm 0.15$ & $7.90 \pm 0.33$ & $7.40 \pm 0.16$ & $7.00 \pm 0.30$ & 0.66 \\
\hline Type-II & $8.10 \pm 0.18$ & $7.80 \pm 0.20$ & $7.70 \pm 0.33$ & $6.90 \pm 0.18$ & $6.90 \pm 0.23$ & 0.63 \\
\hline Type-III & $8.40 \pm 0.27$ & $7.90 \pm 0.18$ & $6.90 \pm 0.41$ & $6.10 \pm 0.18$ & $6.10 \pm 0.10$ & 0.71 \\
\hline \multicolumn{7}{|c|}{ Overall acceptability } \\
\hline Control & $8.16 \pm 0.17$ & $8.06 \pm 0.08$ & $7.84 \pm 0.15$ & $7.70 \pm 0.14$ & $6.74 \pm 0.10$ & 0.38 \\
\hline Type-I & $8.22 \pm 0.12$ & $8.12 \pm 0.09$ & $8.20 \pm 0.25$ & $7.46 \pm 0.10$ & $6.82 \pm 0.10$ & 0.42 \\
\hline Type-II & $8.02 \pm 0.08$ & $7.98 \pm 0.07$ & $8.18 \pm 0.26$ & $7.12 \pm 0.10$ & $7.08 \pm 0.10$ & 0.39 \\
\hline Type-III & $8.30 \pm 0.22$ & $8.06 \pm 0.13$ & $7.56 \pm 0.22$ & $6.42 \pm 0.17$ & $6.54 \pm 0.10$ & 0.51 \\
\hline
\end{tabular}

Values are mean \pm SE of ten observations.

Control= RWF: GPSF:: 100:0, Type-I=RWF: GPSF:: 90:10, Type-II=RWF: GPSF:: 80:20,

Type-III=RWF: GPSF:: 70:30, RWF: Refined wheat flour, GPSF: Germinated pumpkin seed flour 
Table.3 Effect of storage on peroxide value (meq peroxide/kg) of developed value added whole wheat flour biscuits (dry weight basis)

\begin{tabular}{|c|c|c|c|c|c|c|}
\hline $\begin{array}{c}\text { Type of whole wheat } \\
\text { flour biscuits }\end{array}$ & \multicolumn{5}{|c|}{ Storage period (days) } \\
\cline { 2 - 7 } & $\mathbf{1 5}$ & $\mathbf{3 0}$ & $\mathbf{4 5}$ & $\mathbf{6 0}$ & $\mathbf{7 5}$ & $\mathbf{C D}(\mathbf{P} \leq \mathbf{0 . 0 5})$ \\
\hline $\begin{array}{c}\text { Control } \\
\text { WWF:GPSF::100:0 }\end{array}$ & $4.60 \pm 0.17$ & $5.17 \pm 0.09$ & $5.50 \pm 0.12$ & $6.17 \pm 0.15$ & $6.73 \pm 0.09$ & 0.28 \\
\hline $\begin{array}{c}\text { Type-I } \\
\text { WWF:GPSF::90:10 }\end{array}$ & $4.27 \pm 0.11$ & $4.73 \pm 0.14$ & $5.18 \pm 0.16$ & $5.77 \pm 0.09$ & $6.33 \pm 0.18$ & 0.42 \\
\hline $\begin{array}{c}\text { Type-II } \\
\text { WWF:GPSF::80:20 }\end{array}$ & $3.20 \pm 0.13$ & $3.70 \pm 0.14$ & $4.20 \pm 0.16$ & $4.69 \pm 0.18$ & $5.03 \pm 0.09$ & 0.47 \\
\hline $\begin{array}{c}\text { Type-III } \\
\text { WWF:GPSF::70:30 }\end{array}$ & $2.81 \pm 0.12$ & $3.13 \pm 0.15$ & $3.63 \pm 0.09$ & $4.13 \pm 0.09$ & $4.47 \pm 0.15$ & 0.31 \\
\hline
\end{tabular}

Values are mean \pm SE of six independent determinations. WWF: Whole wheat flour, GPSF: Germinated pumpkin seed flour

Table.4 Effect of storage on peroxide value (meq peroxide/1000g) of developed value added cookies (dry weight basis)

\begin{tabular}{|c|c|c|c|c|c|c|}
\hline Types of cookies & \multicolumn{5}{|c|}{ Storage period (days) } \\
\cline { 2 - 7 } & $\mathbf{1 5}$ & $\mathbf{3 0}$ & $\mathbf{4 5}$ & $\mathbf{6 0}$ & $\mathbf{7 5}$ & $\mathbf{C D}(\mathbf{P} \leq \mathbf{0 . 0 5})$ \\
\hline $\begin{array}{c}\text { Control } \\
\text { RWF:GPSF::100:0 }\end{array}$ & $3.20 \pm 0.12$ & $3.63 \pm 0.09$ & $4.20 \pm 0.18$ & $4.59 \pm 0.15$ & $5.00 \pm 0.14$ & 0.38 \\
\hline $\begin{array}{c}\text { Type-I } \\
\text { RWF:GPSF::90:10 }\end{array}$ & $2.40 \pm 0.17$ & $3.30 \pm 0.12$ & $3.40 \pm 0.12$ & $4.27 \pm 0.13$ & $4.60 \pm 0.12$ & 0.17 \\
\hline $\begin{array}{c}\text { Type-II } \\
\text { RWF:GPSF::80:20 }\end{array}$ & $2.27 \pm 0.15$ & $2.70 \pm 0.16$ & $3.10 \pm 0.10$ & $3.60 \pm 0.12$ & $4.13 \pm 0.09$ & 0.24 \\
\hline $\begin{array}{c}\text { Type-III } \\
\text { RWF:GPSF::70:30 }\end{array}$ & $1.80 \pm 0.06$ & $2.40 \pm 0.13$ & $2.70 \pm 0.11$ & $3.30 \pm 0.06$ & $3.70 \pm 0.12$ & 0.39 \\
\hline
\end{tabular}

Values are mean \pm SE of six independent determinations. RWF: Refined wheat flour, GPSF: Germinated pumpkin seed flour

Table.5 Total plate count (log cfu/g) of developed value added whole wheat flour biscuit during storage

\begin{tabular}{|c|c|c|c|c|c|c|}
\hline Types of biscuits & \multicolumn{5}{|c|}{ Storage period (days) } \\
\cline { 2 - 7 } & $\mathbf{1 5}$ & $\mathbf{3 0}$ & $\mathbf{4 5}$ & $\mathbf{6 0}$ & $\mathbf{7 5}$ & $\begin{array}{c}\text { CD } \\
(\mathbf{P} \leq \mathbf{0 . 0 5})\end{array}$ \\
\hline $\begin{array}{c}\text { Control } \\
\text { WWF:GPSF::100:0 }\end{array}$ & $3.20 \pm 0.03$ & $3.51 \pm 0.06$ & $3.81 \pm 0.08$ & $4.11 \pm 0.17$ & $4.32 \pm 0.29$ & 0.17 \\
\hline $\begin{array}{c}\text { Type-I } \\
\text { WWF:GPSF::90:10 }\end{array}$ & $3.44 \pm 0.06$ & $3.73 \pm 0.09$ & $3.99 \pm 0.12$ & $4.12 \pm 0.14$ & $4.25 \pm 0.23$ & 0.21 \\
\hline $\begin{array}{c}\text { Type-II } \\
\text { WWF:GPSF::80:20 }\end{array}$ & $3.55 \pm 0.08$ & $3.85 \pm 0.11$ & $4.11 \pm 0.16$ & $4.15 \pm 0.20$ & $4.20 \pm 0.18$ & 0.12 \\
\hline $\begin{array}{c}\text { Type-III } \\
\text { WWF:GPSF::70:30 }\end{array}$ & $3.67 \pm 0.10$ & $3.99 \pm 0.12$ & $4.04 \pm 0.13$ & $4.16 \pm 0.21$ & $4.21 \pm 0.22$ & 0.03 \\
\hline Vat
\end{tabular}

Values are mean \pm SE of three independent determinations. WWF: Whole wheat flour, GPSF: Germinated pumpkin seed flour 
Table.6 Total plate count (log cfu/g) of developed value added cookies during storage

\begin{tabular}{|c|c|c|c|c|c|c|}
\hline Types of cookies & \multicolumn{5}{|c|}{ Storage period (days) } \\
\cline { 2 - 7 } & $\mathbf{1 5}$ & $\mathbf{3 0}$ & $\mathbf{4 5}$ & $\mathbf{6 0}$ & $\mathbf{7 5}$ & $\begin{array}{c}\text { C.D } \\
(\mathbf{P} \leq \mathbf{0 . 0 5})\end{array}$ \\
\hline $\begin{array}{c}\text { Control } \\
\text { RWF:GPSF::100:0 }\end{array}$ & $3.33 \pm 0.13$ & $3.65 \pm 0.15$ & $3.82 \pm 0.16$ & $3.95 \pm 0.17$ & $4.25 \pm 0.18$ & 0.19 \\
\hline $\begin{array}{c}\text { Type-I } \\
\text { RWF:GPSF::90:10 }\end{array}$ & $3.23 \pm 0.16$ & $3.57 \pm 0.05$ & $3.67 \pm 0.05$ & $3.78 \pm 0.07$ & $4.15 \pm 0.11$ & 0.12 \\
\hline $\begin{array}{c}\text { Type-II } \\
\text { RWF:GPSF::80:20 }\end{array}$ & $3.19 \pm 0.14$ & $3.55 \pm 0.11$ & $3.69 \pm 0.13$ & $3.70 \pm 0.16$ & $4.12 \pm 0.19$ & 0.31 \\
\hline $\begin{array}{c}\text { Type-III } \\
\text { RWF:GPSF::70:30 }\end{array}$ & $3.16 \pm 0.16$ & $3.51 \pm 0.08$ & $3.60 \pm 0.10$ & $3.71 \pm 0.12$ & $4.04 \pm 0.21$ & 0.08 \\
\hline Vatus
\end{tabular}

Values are mean \pm SE of three independent determinations. RWF: Refined wheat flour, GPSF: Germinated pumpkin seed flour

\section{Total plate count}

The total plate count of control whole wheat flour biscuits varied from 3.20 to $4.32 \mathrm{log}$ cfu/g during 75 days of storage. The total plate count of Type-I and Type-II whole wheat flour biscuits ranged from 3.44 to 4.25 $\log \mathrm{cfu} / \mathrm{g}$ and 3.55 to $4.20 \mathrm{log} \mathrm{cfu} / \mathrm{g}$, respectively while that of Type-III whole wheat flour biscuits ranged from 3.67 to 4.21 $\log \mathrm{cfu} / \mathrm{g}$ of biscuits. Total plate count increased significantly $(\mathrm{P} \leq 0.05)$ with the increase in period of storage.

The total plate count of control refined wheat flour cookies varied from 3.33 to $4.25 \mathrm{log}$ cfu/g from day 15 to 75 (Table 6). The total plate count of Type-I and Type-II refined wheat flour cookies ranged from 3.23 to 4.15 $\log \mathrm{cfu} / \mathrm{g}$ and 3.19 to $4.12 \log \mathrm{cfu} / \mathrm{g}$, respectively while that of Type-III refined wheat flour cookies varied from 3.16 to 4.04 $\log \mathrm{cfu} / \mathrm{g}$ of cookies.

Similar work on shelf life of value added products has been reported by a number of coworkers (Peter-Ikechukwu et al., 2018; Goyat et al., 2018). Thivani et al., 2016reported development of wheat flour biscuits incorporated with pineapple powder at the rates of $3,5,10$, and $15 \%$ (w/w basis). They reported that the biscuits prepared with $5 \%$ pineapple powder had the highest nutritional and sensory quality, having the overall acceptability score of 7.7 in a 9-point hedonic scale. The shelf life evaluation showed that these biscuits could be stored for 6 weeks at the ambient conditions of average temperature at $30 \pm 1^{\circ} \mathrm{C}$ and $\mathrm{RH}$ at $75-80 \%$ with acceptable quality. Duta et al., (2019) studied quality of gluten-free oat biscuits for a storage period up to 3 months in different packages. The highest peroxide value was obtained for biscuits packed in PVC_12, while the lowest was for the PE/EVOH/PP_50, for both storage conditions. The biscuits' colour changed from yellow-brown to light yellow and the change was more pronounced in the light as compared to the dark storage conditions. The electronic nose system showed that the distinct volatile composition of the biscuits stored in the light was correlated with the higher scores of the off-flavour attribute and with the peroxide values.

In conclusion the value added cookies and whole wheat flour biscuits with 30 per cent incorporation of germinated pumpkin seed flour could successfully be stored upto 60 and 
75 days respectively. The developed cookies and biscuits therefore have the potential for commercialization. Different packaging materials need to be explored to retain the health benefits of such products.

\section{References}

AOAC. 2010. Official methods of analysis. Association of Official Analytical Chemist. Washington, D. C.

APHA. 1980. Compendium of methods for the microbiological examination of food. $2^{\text {nd }}$ edn., American Public Health Association, Washington D.C.

Duta, D. E., Culetu, A. and Mohan, G. 2019. Sensory and physicochemical changes in gluten-free oat biscuits stored under different packaging and light conditions. Journal of food science and technology, 56(8), 3823-3835.

Goyat, J., Passi, S. J., Suri, S. and Dutta, H. 2018. Development of chia (Salvia hispanica, L.) and quinoa (Chenopodium quinoa, L.) seed flour substituted cookies-physicochemical, nutritional and storage studies. Current Research in Nutrition and Food Science Journal, 6(3), 757-769.

Huang, X. E., Hirose, K., Wakai, K., Matsuo, K., Ito, H., Xiang, J., Takezaki, T. and Tajima, K. 2004. Comparison of lifestyle risk factors by family history for gastric, breast, lung and colorectal. Asian Pacific Journal of Cancer Prevention. 5(4): 419-427.

Karrar, E., Sheth, S., Navicha, W. B., Wei, W., Hassanin, H., Abdalla, M., \& Wang, X. 2019. A potential new source: Nutritional and antioxidant properties of edible oils from cucurbit seeds and their impact on human health. Journal of food biochemistry, 43(2), e12733.

Khairi, N. A., Gobouri, A. A. and Dhahawi, H. O. 2014. A review on omega-3 and omega-6 essential fatty acids: uses, benefits and their availability in pumpkins (Cucurbita maxima) seed and desert dates (Balanitesa egyptiaca) seed kernel oils. Pakistan Journal of Biological Sciences. 17 (12): 11951208.

Kumari, N. and Sindhu, S. C. 2019. Nutrient and mineral composition of developed value added cookies incorporating germinated pumpkin seed powder. IJCS, 7(3), 4583-4586.

Kumari, N., Sindhu, S. C., Kumari, V. and Rani, V. 2020. Nutritional evaluation of developed value-added biscuits incorporating germinated pumpkin seed flour. Journal of Pharmacognosy and Phytochemistry, 9(5), 2802-2806.

Lestari, B. and Meiyanto, E. 2018. A review: The emerging nutraceutical potential of pumpkin seeds. Indonesian Journal of Cancer Chemoprevention, 9(2), 92-101.

Maheshwari, P., Prasad, N. and Batra, E. 2015. Papitas -the underutilized by product and the future cash crop- a review. American International Journal of Research in Formal, Applied and Natural Sciences. 12 (1): 31-34.

Montesano, D., Rocchetti, G., Putnik, P. and Lucini, L. 2018. Bioactive profile of pumpkin: An overview on terpenoids and their health-promoting properties. Current Opinion in Food Science, 22, 81-87.

Nyam, K. L., Lau, M. and Tan, C. P. 2013. Fibre from pumpkin (Cucurbita pepo L.) seeds and rinds: physio-chemical properties, antioxidant capacity and application as bakery product ingredients. Malaysian Journal of Nutrition. 19: 99-109.

Patel, S., \& Rauf, A. 2017. Edible seeds from Cucurbitaceae family as potential functional foods: Immense promises, few concerns. Biomedicine \& Pharmacotherapy, 91, 330-337. 
Peter-Ikechukwu, A. I., Omeire, G. C., Kabuo, N. O., Eluchie, C. N., Amandikwa, C. and Odoemenam, G. I. 2018. Production and evaluation of biscuits made from wheat Flour and toasted watermelon seed meal as fat substitute. Journal of Food Research, 7(5), 112-123.
Thivani, M., Mahendren, T. and Kanimoly, M. 2016. Study on the physicochemical properties, sensory attributes and shelf life of pineapple powder incorporated biscuits. Ruhuna Journal of Science, 7(2 December).

\section{How to cite this article:}

Neeta Kumari, Sangeeta C. Sindhu, Varsha Rani and Varsha Kumari. 2021. Shelf Life Evaluation of Biscuits and Cookies Incorporating Germinated Pumpkin Seed Flour. Int.J.Curr.Microbiol.App.Sci. 10(01): 1436-1443.

doi: https://doi.org/10.20546/ijcmas.2021.1001.170 